\title{
Resistance to antimicrobial drugs in Ghana
}

This article was published in the following Dove Press journal:

Infection and Drug Resistance

6 December 201I

Number of times this article has been viewed

Mercy J Newman'

Enoch Frimpong ${ }^{2}$

Eric S Donkor'

Japheth A Opintan'

Alex Asamoah-Adu ${ }^{3}$

'Department of Microbiology,

University of Ghana Medical School,

Accra, Ghana, ${ }^{2}$ School of Medical

Sciences, Kwame Nkrumah University

of Science and Technology, ${ }^{3}$ Public

Health Reference Laboratory,

Korle-Bu, Accra, Ghana
Correspondence: Eric S Donkor

Department of Microbiology,

University of Ghana Medical School,

Box 4236, Accra, Ghana

$\mathrm{Tel}+233302665404$

Fax +233302663062

Email ericsdon@hotmail.com
Background: Antimicrobial drug resistance is a global issue that affects health, economic, and social development. The problem has been attributed to misuse of antimicrobial agents.

Purpose: To identify the agents of bacterial infection in Ghana, determine their antibiogram, and the possibility of setting up a surveillance program.

Patients and methods: A prospective quantitative study set in various hospitals including two teaching hospitals, seven regional hospitals, and two district hospitals in Ghana. A total of 5099 bacterial isolates from various clinical specimens were collected over a period of 1 year, including data related to the patients. Susceptibility of the isolates was determined by the KirbyBauer method. In addition, the minimum inhibitory concentration (MIC) of multidrug-resistant isolates of epidemiological significance was also determined using the E-test.

Results: A wide range of bacterial isolates were identified in both teaching and regional hospitals. High percentage of resistance was observed for tetracycline (82\%), cotrimoxazole (73\%), ampicillin (76\%), and chloramphenicol (75\%). Multidrug resistance was observed to a combination of ampicillin, tetracycline, chloramphenicol, and cotrimoxazole. On the other hand, a lower percentage of resistance was observed for ceftriaxone (6.3\%), ciprofloxacin (11\%), and amikacin (9.9\%).

Conclusion: Generally, the prevalence of multidrug resistance was widespread among the various isolates. Some multidrug-resistant strains of Staphylococcus aureus, Salmonella typhi, and non-typhoidal Salmonella (NTS) had high MIC to cefuroxime $(>256)$, gentamicin $(>256)$, and ciprofloxacin $(>32)$.

Keywords: antimicrobial agents, multidrug resistance, MIC

\section{Introduction}

Bacterial infections constitute an important cause of morbidity and mortality among humans all over the world. For decades, antimicrobial drugs have proven useful for the treatment of bacterial infections. Although some bacteria are inherently resistant to even newly developed antimicrobial agents, the emergence of acquired resistance to antimicrobial drugs has been observed in almost all pathogenic bacteria. ${ }^{1,2}$ Antimicrobial resistance has become an important public health problem associated with serious consequences for the treatment of infection. This ultimately affects both economic and social development. The problem has been attributed to the misuse of antimicrobial drugs, which provide selective pressure, favoring the emergence of resistant strains. To contain the problem of antimicrobial resistance, the World Health Organization has provided some interventions. These include creating a national task force, developing indicators to monitor and evaluate the impact of antimicrobial resistance, 
and designing reference microbiological facilities that would coordinate effective surveillance of antimicrobial resistance among common pathogens. ${ }^{3}$ While these interventions seem to have been well implemented in the developed world, lack of resources constrain implementation in many developing countries where treatment options also tend to be relatively limited. Thus, although a global problem, antimicrobial resistance tends to be more significant in developing countries than in the developed world.

Several studies in African countries have reported the presence of resistant strains of bacteria, including reports from Nigeria, ${ }^{4}$ Uganda, ${ }^{5}$ Tanzania,${ }^{6}$ Zimbabwe,${ }^{7}$ and Ghana, ${ }^{8-14}$ all showing high levels of resistance to antimicrobial agents. Most of these reports show bacterial resistance to commonly utilized and relatively cheap drugs like ampicillin, tetracycline, and cotrimoxazole, which have been the mainstay of antimicrobial treatment in Africa for decades.

In Ghana, antimicrobial therapy constitutes a major form of treatment. It is mainly empirical due to a relative lack of appropriate laboratory facilities for culture and susceptibility testing of bacteria in several health facilities. Even where laboratory facilities are available, culture and susceptibility tests may not be requested due to the fact that this is an extra cost to be paid by the patient. In Ghana, drug resistance has mainly been reported from the two teaching hospitals in Accra and Kumasi, Korle-Bu (KBTH) ${ }^{8-11}$ and Komfo Anokye (KATH), ${ }^{12,13}$ respectively, and hardly any from regional and district hospitals. Thus, there is inadequate information on the susceptibility of microorganisms to antimicrobial agents used for treatment in several areas in Ghana. Syndromic treatment has been established for infections such as sexually transmitted diseases and respiratory tract infections. Without surveillance records of antimicrobial susceptibility, empirical treatment could be ineffective and expensive. The objectives of this study were to identify the agents of bacterial infections, the prevalence and trend of bacterial drug resistance, and assess the possibility of setting up a laboratory-based surveillance program in Ghana.

\section{Material and methods Study area and collection of bacteria isolates}

The study was carried out in eleven hospitals and covered nine of the ten regions in Ghana; the study hospitals included two teaching hospitals, seven regional hospitals, and two district hospitals. Arrangements were made with the study hospitals for collection and storage of bacteria isolated from clinical specimens of patients, including cerebrospinal fluid, blood, urine, wound swabs, sputum, and high vaginal swabs. Fastidious bacteria were stored in Trypticase soy broth with glycerol at $4^{\circ} \mathrm{C}$, while non-fastidious bacteria were stored on nutrient agar slopes at $4^{\circ} \mathrm{C}$. The stored isolates were transported at regular intervals to $\mathrm{KBTH}$ in Accra or KATH in Kumasi for analysis. In KBTH and KATH, isolates were tested by standard procedures, and those collected from the other hospitals were retested by standard procedures at KBTH. Over a period of 1 year (December 2002-December 2003) a total of 5099 isolates from various clinical specimens were collected from the hospitals.

\section{Laboratory analysis}

Laboratory tests on isolates involved biochemical identification and susceptibility testing. Firstly, the isolates were identified by culture and biochemical tests. Following this, the Kirby-Bauer method of susceptibility testing ${ }^{15}$ was employed to determine the antibiogram of the isolates using the following 16 antimicrobial drugs with their disk content in micrograms $(\mu \mathrm{g})$ : ampicillin (10), tetracycline (30), cotrimoxazole (25), chloramphenicol (30), gentamicin (30), amikacin (30), cefuroxime (30), cefotaxime (30), ceftriaxone (30), penicillin (10), erythromycin (15), cloxacillin (5), ciprofloxacin (5), norfloxacin (10), nalidixic acid (30), and nitrofurantoin (300), all commonly used in Ghana. Additionally, the E-test was used to determine the MIC of selected isolates identified as multidrug resistant. Such bacteria included S. aureus, S. typhi, and non-typhoidal Salmonella (NTS). The minimum inhibitory concentrations (MICs) were presented as the minimum inhibition concentration for $50 \%$ and $90 \%$ of isolates, $\mathrm{MIC}_{50}$ and $\mathrm{MIC}_{90}$, respectively. The commercial antimicrobial discs and E-strips used were purchased from Oxoid Ltd (Basingstoke, UK) and AB Biodisk (Solna, Sweden), respectively.

\section{Data analysis}

Laboratory results and data collected on patients were entered into Microsoft Excel and analyzed in Stata (v 7.0; Stata Corp, College Station, TX) to address the objectives of the study. Frequencies and prevalence rates were calculated for resistant and multidrug-resistant bacteria agents, including their antibiogram, and comparisons were made between teaching and regional hospitals.

\section{Results}

From December 2002 to December 2003, a total of 5099 isolates from various clinical specimens were collected 
from the hospitals and comprised mainly Gram-negative organisms. The most prevalent organism in the collection was Escherichia coli, followed by S. aureus, Klebsiella spp, and Pseudomonas aeruginosa. These four organisms made up $56 \%$ of the organisms studied, as listed in Table 1 . Most of the bacteria from the regions were of the enterobacteria group, eg, E. coli and Klebsiella ssp., in addition to Pseudomonas and $S$. aureus. A more varied group of organisms were isolated from the teaching hospitals, where the laboratories were able to grow fastidious microorganisms such as Haemophilus, Neisseria, and Streptococcus pneumoniae. For most of the fastidious organisms, the number of isolates studied was relatively few, and no anaerobic bacteria were collected.

Figure 1 shows the overall prevalence of resistance to the antimicrobial agents tested. There was very high resistance to cotrimoxazole (73\%), chloramphenicol (75\%), ampicillin $(76 \%)$, and tetracycline $(82 \%)$. The teaching and regional hospitals presented a mixed percentage prevalence of isolates resistant to the different antimicrobials tested (Figure 2). The overall resistance of some older drugs like tetracycline, cotrimoxazole, ampicillin, and chloramphenicol was higher in the regional hospitals compared to the teaching hospitals $(P>0.05)$. On the other hand, the prevalence of isolates resistant to the quinolones, penicillin, cloxacillin, and erythromycin, was higher in the teaching hospitals compared with the regional hospitals ( $P>0.05$; Figure 2$)$.

Table 2 shows the prevalence of multidrug-resistant strains of different types of bacteria, including E. coli $(69.5 \%)$,

Table I Bacterial isolates obtained from teaching, regional and district hospitals from Ghana $(n=5099)$

\begin{tabular}{ll}
\hline Bacterial agent & $\mathbf{n}(\%)$ \\
\hline Escherichia coli & $\mathrm{I} I 05(2 \mathrm{I} .7)$ \\
Staphylococcus aureus & $788(\mathrm{I} 5.5)$ \\
Klebsiella spp & $536(10.5)$ \\
Pseudomonas aeruginosa & $44 \mathrm{I}(8.7)$ \\
Proteus spp & $397(7.8)$ \\
Enterobacter spp & $275(5.4)$ \\
Non-typhoidal Salmonella & $247(4.8)$ \\
Other Streptococcus spp & $127(2.5)$ \\
Citrobacter spp & $120(2.4)$ \\
Salmonella typhi & $109(2.1)$ \\
Acinetobacter spp & $88(\mathrm{I} .7)$ \\
Streptococcus pneumoniae & $5 \mathrm{I}(\mathrm{I} .0)$ \\
Neisseria gonorrhoeae & $\mathrm{I} 7(0.3)$ \\
Neisseria meningitidis & $\mathrm{II}(0.02)$ \\
Haemophilus influenzae & $4(0.08)$ \\
Shigella spp & $4(0.08)$ \\
Vibrio cholerae & $\mathrm{I}(0.02)$ \\
Others & $778(\mathrm{I} 5.4)$ \\
\hline
\end{tabular}

Enterobacter species (60.4\%), S. typhi (62.4\%), Citrobacter (65\%), and S. aureus (42.3\%). Table 3 summarizes the $\mathrm{MIC}_{50}$ and $\mathrm{MIC}_{90}$ values of $S$. aureus, S. typhi, and NTS to selected antimicrobials. Full resistance to ciprofloxacin was not observed for S. typhi and NTS in the isolates tested. However, for cefuroxime and gentamicin, the $\mathrm{MIC}_{90}$ were generally high. For example, the $\mathrm{MIC}_{90}$ for $S$. aureus, S. typhi, and NTS were all $>256 \mu \mathrm{g} / \mathrm{mL}^{-1}$ (Table 3).

The biochemical identification of the organisms collected from the regions was comparable to those retested in KBTH. Out of a total of 387 isolates from nonteaching or regional and district hospitals, 150 (39\%) had similar susceptibility results, but the others showed discrepancies ranging from $53 \%$ to $75 \%$.

\section{Discussion}

Bacteria causing infections from various specimens in Ghana were identified as including E. coli, S. aureus, Klebsiella, Pseudomonas, and Salmonella species. Most of the isolates from the regions were Gram-negative rods, eg, enterobacteria and Pseudomonas. The fastidious organisms like Streptococcus pneumoniae, Neisseria gonorrhoeae, and Haemophilus species were isolated only from the teaching hospitals. The inability to isolate fastidious organisms in some of the hospitals was due to lack of particular or special media and other requirements for culture and identification, or due to lack of technical expertise.

The prevalence of drug resistance among the bacteria was high. Four antimicrobial agents had very high prevalence of resistance, namely tetracycline (82\%), ampicillin $(76 \%)$, chloramphenicol (75\%), and cotrimoxazole (73\%), and for these drugs, the prevalence of resistance is higher in the regions than in the teaching hospitals. In addition, these are drugs that have been reported as having high-percentage resistance for a number of microorganisms for several years. ${ }^{16,17}$ The rate of resistance had been rising over the years, not only for clinical isolates but also for the normal intestinal flora of the healthy population. ${ }^{16}$ Lower prevalence of resistance was found for gentamicin (28\%), cefuroxime (27\%), cefotaxime, and norfloxacin (20\%). The lowest prevalence of resistance of between $6 \%-10 \%$ was found in ciprofloxacin, amikacin, and ceftriaxone. These are drugs which have been on the Ghanaian market for a relatively short period of time compared to drugs like ampicillin and chloramphenicol. In addition, ciprofloxacin, amikacin, and ceftriaxone are very expensive drugs and are usually prescribed for serious infections. This might be one of the reasons for the relatively low levels of resistance. 


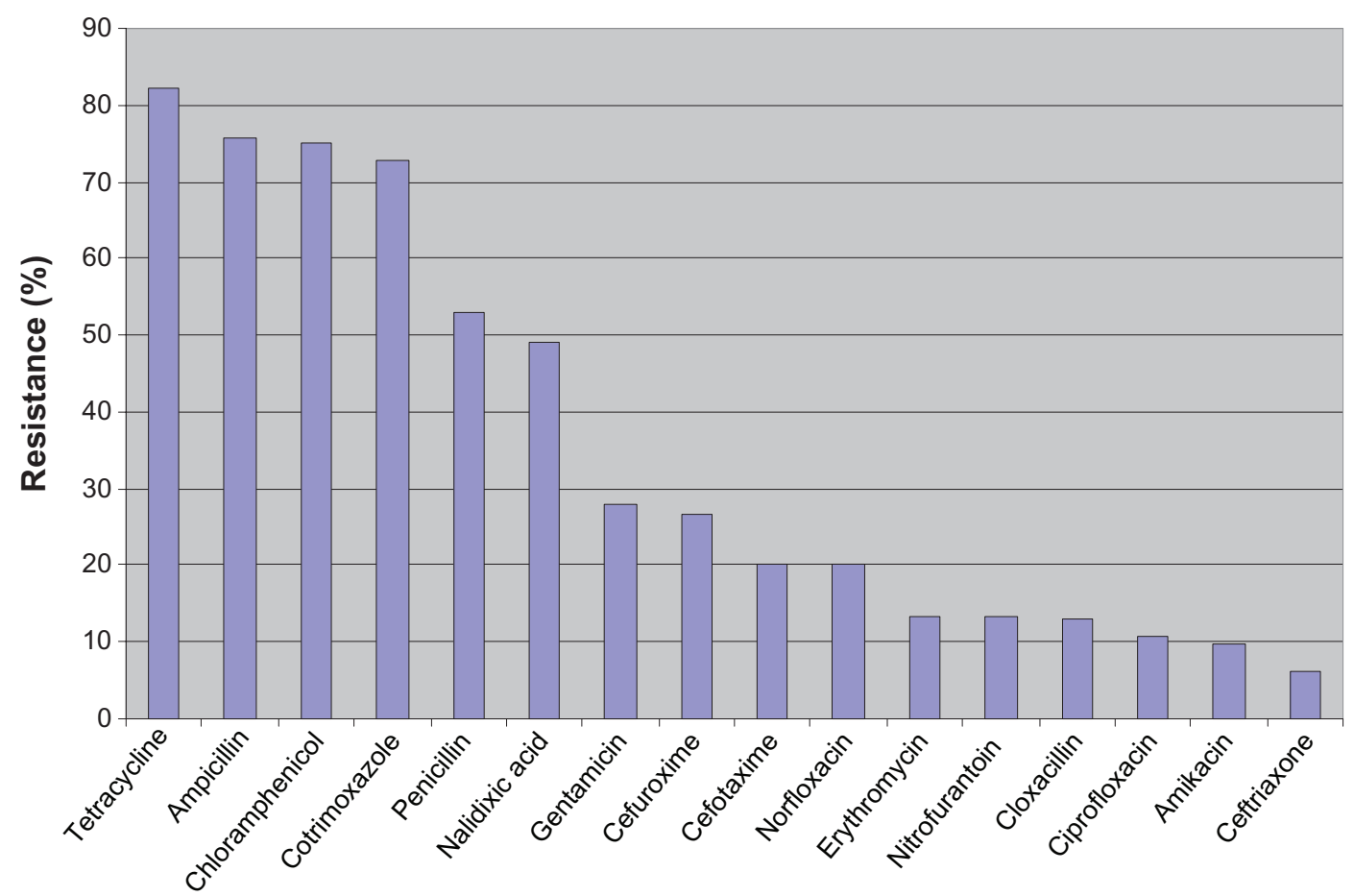

Antimicrobial drug

Figure I Prevalence of resistance among antimicrobial drugs from teaching, regional, and district hospitals in Ghana $(n=5099)$.

The resistant profiles observed in this study are similar to those reported by a relatively recent study in $2008 .{ }^{14}$ In that study, the authors observed low prevalence of resistance to ciprofloxacin, amikacin, and ceftriaxone, as in the current study. This suggests that the resistance profiles of these drugs have not changed significantly over the 5-year period between the two studies. It must be noted, however, that resistance to the fluoroquinolones, such as ciprofloxacin and ofloxacin, has been rising in several countries. ${ }^{18}$ Recently, the mechanism of quinolone resistance has been shown to be associated with upregulation of efflux pumps, which export quinolones and other antimicrobials out of the bacterial cell. ${ }^{19}$ Additionally, mutations in the quinolone resistance-determining regions (QRDRs) of gyrA and parC are also common resistance mechanisms indicating that resistance to one fluoroquinolone may extend to other fluoroquinolones. ${ }^{19,20}$ It is therefore necessary to control the use of these agents so as to maintain their effectiveness for a longer period.

Resistance to antimicrobial agents is a bacteria characteristic carried on genes. The resistance genes present

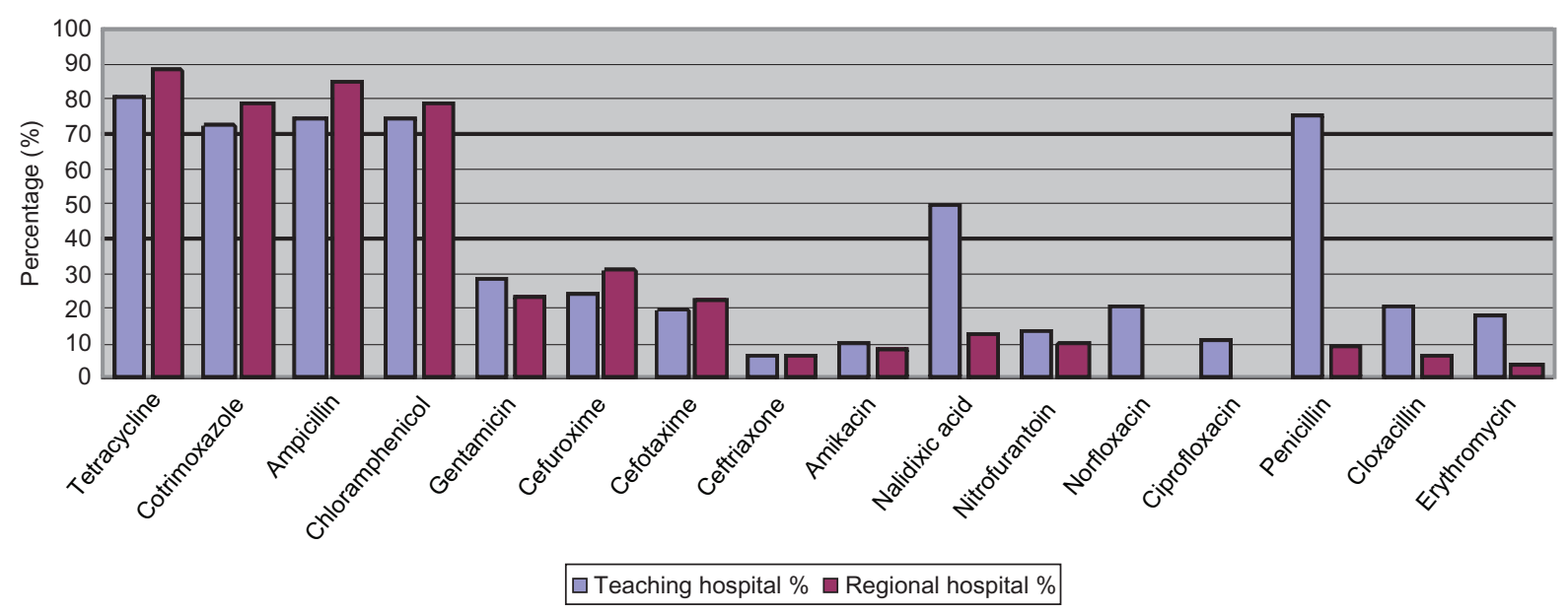

Figure 2 Prevalence of resistance among antimicrobial drugs in teaching $(n=4527)$ and regional hospitals $(n=763)$ in Ghana. 
Table 2 Prevalence of multidrug resistance among bacterial agents isolated from teaching, regional and district hospitals in Ghana

\begin{tabular}{lll}
\hline Bacteria & Number & $\begin{array}{l}\text { Multidrug-resistant } \\
\text { isolates n (\%) }\end{array}$ \\
\hline Pseudomonas aeruginosa & 441 & $100(22.7)$ \\
Other Streptococcus spp & 127 & $100(78.7)$ \\
Acinetobacter spp & 88 & $57(64.8)$ \\
Citrobacter spp & 120 & $78(65.0)$ \\
Streptococcus pneumoniae & 51 & $4(7.8)$ \\
Escherichia coli & 1105 & $768(69.5)$ \\
Enterobacter spp & 275 & $166(60.4)$ \\
Salmonella typhi & 109 & $68(62.4)$ \\
Non-typhoidal Salmonella & 247 & $149(60.3)$ \\
Klebsiella spp & 536 & $309(57.6)$ \\
Proteus spp & 397 & $222(55.9)$ \\
Neisseria gonorrhoeae & 17 & $2(11.8)$ \\
Staphylococcus aureus & 788 & $333(42.3)$ \\
\hline
\end{tabular}

in intestinal flora are transferable to pathogenic bacteria in the intestines. Plasmid-mediated resistance is more easily transferred than chromosomal genes. Mills-Robertson et al reported the location of plasmids in Salmonella on conjugative plasmids. ${ }^{21}$ The plasmids could have evolved because of antibiotic misuse. There is antibiotic misuse by the general public due to easy availability of drugs without prescription, or by physicians, through poor prescribing habits.

This study did not differentiate between organisms from community-acquired infections and those from hospital infections. A study in Kenya showed that organisms from the community and those from hospital infections had the same patterns of multidrug resistance. ${ }^{22}$ It may be necessary in future to compare the susceptibility of community and hospital isolates of bacteria.

Table 3 Minimum inhibitory concentration $(\mu \mathrm{g} / \mathrm{mL})$ of selected isolates for cefuroxime, gentamicin and ciprofloxacin

\begin{tabular}{lclll}
\hline $\begin{array}{l}\text { Organism/ } \\
\text { antimicrobial agent }\end{array}$ & $\mathbf{n}$ & $\begin{array}{l}\text { MIC range } \\
\mu \mathrm{g} / \mathbf{m L}\end{array}$ & MIC $_{50}$ & MIC $_{90}$ \\
\hline $\begin{array}{l}\text { Staphylococcus aureus } \\
\quad \text { Cefuroxime }\end{array}$ & 18 & & & \\
$\quad$ Gentamicin & & $0.25-256$ & 4.0 & $>256$ \\
Salmonella typhi & 10 & & 2.0 & $>256$ \\
$\quad$ Cefuroxime & & $1.5-256$ & 3.0 & $>256$ \\
$\quad$ Gentamicin & & $0.19-1.5$ & 0.38 & 0.75 \\
$\quad$ Ciprofloxacin & & $0.004-0.094$ & 0.016 & 0.064 \\
Non-typhoidal Salmonella & 14 & & & \\
$\quad$ Cefuroxime & & $3.0-256$ & 4.0 & $>256$ \\
$\quad$ Gentamicin & & $0.25-4.0$ & 0.75 & 3.0 \\
$\quad$ Ciprofloxacin & & $0.004-32$ & 0.012 & 0.25 \\
\hline
\end{tabular}

Note: Disc concentration $(\mu \mathrm{g} / \mathrm{mL})$ of E-strips of cefuroxime, gentamicin and ciprofloxacin used were $0.023-256,0.023-256$ and $0.002-32$, respectively.

Abbreviation: $\mathrm{MIC}_{50}$ and $\mathrm{MIC}_{90}$, minimum inhibition concentration for $50 \%$ and $90 \%$ of the isolates, respectively.
In view of the low numbers of some organisms like Haemophilus, Neisseria, and Vibrio species, it was difficult to comment adequately on their findings. It may, therefore, be necessary to collect more data in future and analyze the results. Although very few isolates of $N$. gonorrhoeae were received, studies in other African countries show that resistant strains are endemic in Africa. ${ }^{23}$ All these studies demonstrate that local surveillance is necessary to help in decision making in relation to syndromic treatment and implementation of control measures. In view of the discrepancies in the susceptibility results from the various hospitals, it will be inappropriate to start collecting susceptibility reports for surveillance purposes. Standardized methods of susceptibility testing must be used in all laboratories before laboratory-based surveillance can be used in Ghana.

In the current study, the $\mathrm{MIC}_{90}$ for cefuroxime and gentamicin against $S$. aureus and $S$. typhi exceeded $256 \mu \mathrm{g} / \mathrm{mL}$. These microorganisms are highly resistant, and this gives cause for concern. Therefore, there is a need for a bigger study of MICs of epidemiologically significant clinical isolates in Ghana.

There are certain limitations to this study. In the period 2002-2003, patients were required to pay for the cost of their investigations; invariably, only a relatively small portion of all cases with an infection had cultures taken to determine the causative agent and its drug susceptibility. In addition, when an infection seemed to be serious, it was more likely to be investigated, especially if the patient was not responding to treatment. Ideally, one needs to investigate many episodes of a particular infection to determine the types of causative agent(s). Anaerobic bacteria were not collected from any of the hospitals. Although KBTH and KATH had trained personnel, they were unable to culture anaerobes. This is due to the fact that it is very expensive to acquire Gas Pak systems to generate an anaerobic environment for the culture of anaerobes.

\section{Acknowledgments}

The authors are grateful to all the collaborating hospitals, especially the technologists and technicians from the respective bacteriology departments. This study was funded by the Ghanaian Dutch Collaboration for Health and Development.

\section{Disclosure}

The authors declare no conflicts of interest in relation to this paper. 


\section{References}

1. Andersson DI, Hughes D. Persistence of antibiotic resistance in bacterial populations. FEMS Microbiol Rev. 2011;35(5):901-911.

2. Planson AG, Carbonell P, Grigoras I, Faulon JL. Engineering antibiotic production and overcoming bacterial resistance. Biotechnol J. 2011; $6(7): 812-825$.

3. World Health Organization. WHO Global Strategy for Containment of Antimicrobial Resistance (WHO/CDC/CSR/DRS/2001.2a). Geneva, Switzerland: World Health Organization; 2001.

4. Dada-Adegbola HO, Muili KA. Antibiotic susceptibility pattern of urinary tract pathogens in Ibadan, Nigeria. Afr J Med Med Sci. 2010; 39(3):173-179.

5. Andabati G, Byamugisha J. Microbial aetiology and sensitivity of asymptomatic bacteriuria among ante-natal mothers in Mulago hospital, Uganda. Afr Health Sci. 2010;10(4):349-352.

6. Moyo S, Aboud S, Kasubi M, Maselle SY. Bacteria isolated from bloodstream infections at a tertiary hospital in Dar es Salaam, Tanzania - antimicrobial resistance of isolates. S Afr Med J. 2010; 100(12):835-838.

7. Mbanga J, Dube S, Munyanduki H. Prevalence and drug resistance in bacteria of the urinary tract infections in Bulawayo province, Zimbabwe. East Afr J Public Health. 2010;7(3):229-232.

8. Newman MJ. Multiple-resistant Salmonella group G outbreak in a neonatal intensive care unit. West Afr J Med. 1996;15(3):165-169.

9. Voros S, Salles CA, Marbell EC, Asamoah-Adu A, Afoakwa SN, Kelemen G. Salmonellosis in Accra. Ghana Med J. 1976;15:109-114.

10. Newman MJ. Antimicrobial resistance of urinary isolates in Accra. Ghana Med J. 1990;24(2):154-158.

11. Opintan JA, Newman MJ. Distribution of serogroups and serotypes of multiple drug resistant Shigella isolates. Ghana Med J. 2007; 41(1):50-54.

12. Ohene A. Bacterial pathogens and their antimicrobial susceptibility in Kumasi, Ghana. East Afr Med J. 1997:74(7):450-455.
13. Danso KA, Adu-Sarkodie Y. Abdominal wound infection complicating caesarean sections. Ghana Med J. 1998;32b:1003-1008.

14. Edoh D, Alomatu B. Comparison of antibiotic resistance patterns between laboratories in Accra east Ghana. Nigerian Annals of Natural Science. 2008:8(1):10-18.

15. Bauer AW, Kirby WM, Sherris JC, Turck M. Antibiotic susceptibility testing by a standardized single disc method. Am J Clin Pathol. 1966; 45(4):493-496.

16. Wilcox MH. The tide of antimicrobial resistance and selection. Int J Antimicrob Agents. 2009;34 Suppl 3:S6-S10.

17. Gould IM. Antibiotic resistance: the perfect storm. Int J Antimicrob Agents. 2009;34 Suppl 3:S2-S5.

18. Gottesman BS, Carmeli Y, Shitrit P, Chowers M. Impact of quinolone restriction on resistance patterns of Escherichia coli isolated from urine by culture in a community setting. Clin Infect Dis. 2009;49(6): 869-875.

19. Wang H, Dzink-Fox JL, Chen M, Levy SB. Genetic characterization of highly fluoroquinolone-resistant clinical Escherichia coli strains from China: role of acrR mutations. Antimicrob Agents Chemother. 2001; 45(5):1515-1521.

20. Namboodiri SS, Opintan JA, Lijek RS, Newman MJ, Okeke IN. Quinolone resistance in Escherichia coli from Accra, Ghana. BMC Microbiol. 2011;11:44.

21. Mills-Robertson F, Crupper SS, Addy ME, Mensah P. Antibiotic resistance and genotyping of clinical group B Salmonella isolated in Accra, Ghana. J Appl Microbiol. 2003;94(2):289-294.

22. Malonza IM, Omari MA, Bwayo JJ, et al. Community acquired bacterial infections and their antimicrobial susceptibility in Nairobi, Kenya. East Afr Med J. 1997;74(3):166-170.

23. Tadesse A, Mekonnen A, Kassu A, Asmelash T. Antimicrobial sensitivity of Neisseria gonorrhoea in Gondar, Ethiopia. East Afr Med J. 2001;78(5):259-261.
Infection and Drug Resistance

\section{Publish your work in this journal}

Infection and Drug Resistance is an international, peer-reviewed openaccess journal that focuses on the optimal treatment of infection (bacterial, fungal and viral) and the development and institution of preventive strategies to minimize the development and spread of resistance. The journal is specifically concerned with the epidemiology of antibiotic

\section{Dovepress}

resistance and the mechanisms of resistance development and diffusion in both hospitals and the community. The manuscript management system is completely online and includes a very quick and fair peerreview system, which is all easy to use. Visit http://www.dovepress.com/ testimonials.php to read real quotes from published authors. 